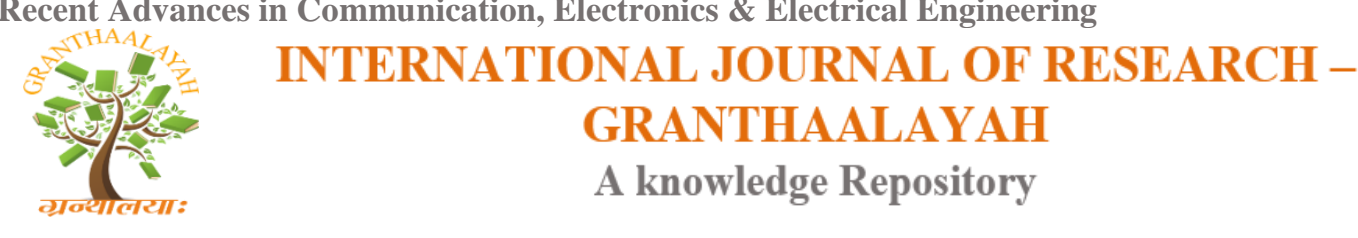

RACEEE - 17

\title{
DIAGNOSIS OF DIABETIC RETINOPATHY THROUGH SCREENING OF RETINAL IMAGES
}

\author{
Purushottama T L ${ }^{* 1}$, Kishore $\mathrm{C}^{2}$ \\ *1,2 Department of Electronics and Communication Engineering, S.I.T, Tumakuru, India
}

DOI: https://doi.org/10.29121/granthaalayah.v5.i4RACEEE.2017.3330

\begin{abstract}
Diabetic Retinopathy (DR) is progressive dysfunction of the retinal blood vessels caused by chronic hyperglycemia which can be a complication of diabetes type 1 or diabetes type 2 . Initially, DR is asymptomatic, if not treated though it can cause low vision and blindness. Diabetic retinopathy is responsible for 1.8 million of the 37 million cases of blindness throughout the world. So the early detection of Diabetic retinopathy through proper screening is essential.

The paper presents a Diabetic Retinopathy Screening System which can be used as a primary diagnosis tool by ophthalmologists in the screening process to detect symptoms of Diabetic Retinopathy. The system uses the anatomical structures such as blood vessels, exudates and microaneurysms in retinal images. The retinal images are segmented and classified as normal or DR affected images by extracting features from segmented images and the Gray Level Cooccurrence Matrix (GLCM). The classifier used is Support Vector Machine (SVM) which gives a better accuracy.

The system is implemented and tested in MATLAB and LabView for the standard database and need to be optimized for real time screening of images. LabView creates distributable .EXE files and .DLL files which can be downloaded into the FPGA/DSP processor. Hardware implementation on LabView FPGA presents a small learning curve which drastically reduces development time and eliminates the need for custom hardware design.
\end{abstract}

Keywords: Diabetic Retinopathy (DR); Blood Vessels; Exudates; Microaneurysms (MA); Morphological Processing; Grey Level Co-Occurrence Matrix (GLCM); Support Vector Machine (SVM).

Cite This Article: Purushottama T L, and Kishore C. (2017). "DIAGNOSIS OF DIABETIC RETINOPATHY THROUGH SCREENING OF RETINAL IMAGES." International Journal of Research - Granthaalayah, 5(4) RACEEE, 92-104. 
[Purushottama et. al., Vol.5 (Iss.4: RACEEE), April, 2017]

ICV (Index Copernicus Value) 2015: 71.21
ISSN- 2350-0530(O), ISSN- 2394-3629(P)

IF: 4.321 (CosmosImpactFactor), 2.532 (I2OR)

InfoBase Index IBI Factor 3.86

Recent Advances in Communication, Electronics \& Electrical Engineering

\section{Introduction}

The main aim of this work is to develop reliable and accurate image processing and pattern recognition methods for automatic fundus image analysis to aid ophthalmologist's diagnosis and to be used as an automatic tool for the screening of diabetic retinopathy. Given a low quality color fundus image, the fundus image analysis system should extract the features such as the optic disk, fovea and the retinal blood vessels as reference coordinates before the system can achieve more complex tasks of identifying pathological entities such as hard exudates, cotton wool spots (soft exudates), hemorrhages and microaneurysms [3]. The system must be able to do it all the time irrespective of variability in color, illumination levels and amount of noise. Finally to develop an algorithm that will be able to identify and classify the patients with normal, moderate and severe from the obtained retinal fundus image of the patients.

\subsection{Diabetic Retinopathy}

Diabetic retinopathy is a complication of diabetes to the retina. It is a very asymptomatic disease in the early stages and it could lead to permanent vision loss if untreated for long time [8]. The problem here is the patients may not know about it until it reaches advanced stages. Once it reaches advanced stages vision loss becomes inevitable. Diabetic retinopathy occurs because of microangiopathy which in turn affects the retinal pre-capillary arterioles, capillaries and venules. It is caused by microvascular leakages from the breakdown of the internal blood-retinal barrier and microvascular occlusion. Due to the progressive damage of the microvascular system, loss of vision and blindness can occur. The presence of diabetic retinopathy can be detected by examining the retina form its characteristic features [9].

\subsection{Abnormalities Associated with the Retina}

Diabetic retinopathy occurs due to the leakage of blood vessels, formation of dark spots on retinal surface, etc. The clinical symptoms of diabetic retinopathy are discussed below. Fig. 1 shows the effect of diabetic retinopathy on vision.

Microaneurysms: These are the first clinical abnormality to be noticed in the eye [8]. They may appear in isolation or in clusters as tiny, dark red spots. Microaneurysms arise due to high sugar levels in the blood which causes the walls of tiny blood vessels to distend. Their sizes ranges from 10-100 microns i.e. less than 1/12th the diameter of an average optics disc and are circular in shape, at this stage, the disease is not eye threatening [1].

Hemorrhages: Occurs in the deeper layers of the retina and are often called 'blot' hemorrhages because of their round shape. As the retinal blood vessels become more damaged and permeable, their number will increase. Retinal hemorrhages look either as small red dots or blots identical to microaneurysms or as larger flame-shaped hemorrhages.

Hard exudates: These are one of the main characteristics of diabetic retinopathy and can vary in size from tiny specks to large patches with clear edges. As well as blood, fluid that is rich in fat and protein is contained in the eye and this is what leaks out to form the exudates. These can impair vision by preventing light from reaching the retina [9]. 
Recent Advances in Communication, Electronics \& Electrical Engineering

Soft exudates: These are often called 'cotton wool spots'. As the disease advances further, multiple small patches of the retina become ischemic deprived of blood. These ischemic regions are observable on the retina as fluffy whitish blobs called cotton wool spots. This is more often seen in advanced retinopathy.

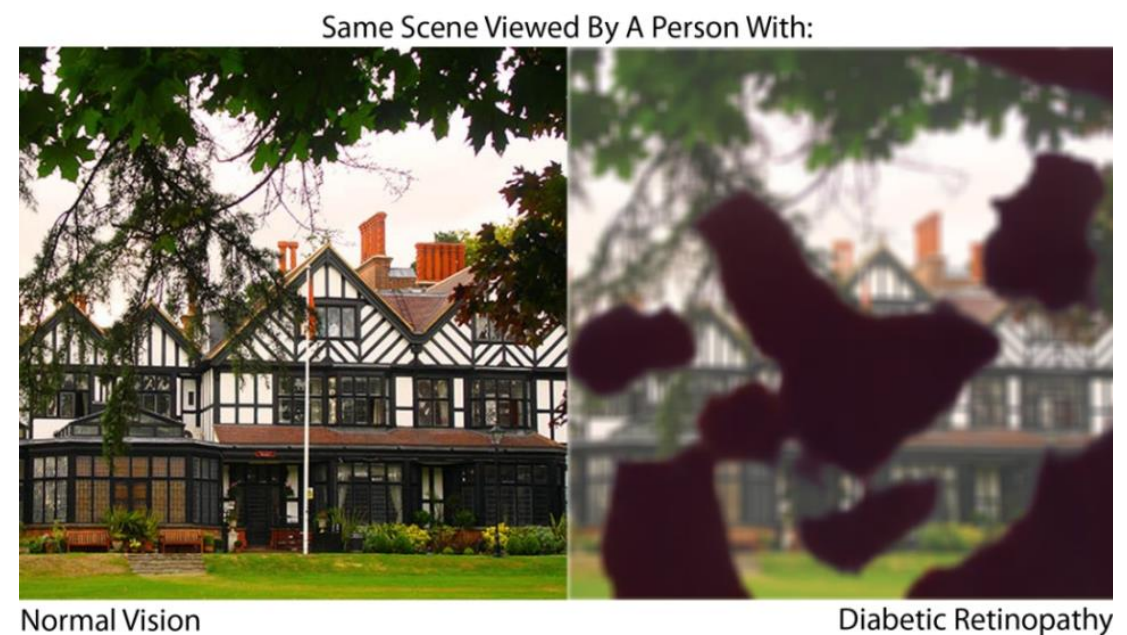

Figure 1: Effect of diabetic retinopathy on vision without retinopathy and with retinopathy

\subsection{Methodologies for Detection and Classification}

The green channel extraction of image gives the better contrast enhancement between foreground and background. Mathematical morphology approach gives the finer details of thin vessels in blood vessel segmentation compared with wavelet transform. K-means clustering is used for exudates segmentation contains some noisy pixel, so it needs to reduce the error due to over enhancement of noise. kNN, NB, and SVM, are used for classification and SVM has shown slightly better result compared to other classifiers.

\section{Methodology}

The proposed automatic screening methodology is shown in Fig. 2. The input to the system retinal fundus image taken by the digital fundus camera. The input retinal images undergo perprocessing to enhance the contrast of an image and segmentation to detect blood vessels, exudates and microaneurysms separately. Textural feature analysis is done by using GLCM. Then features such as area of blood vessels, area of exudates, and area of microaneurysms, energy, entropy, contrast and homogeneity are extracted. These features are fed to SVM classifier which classifies the image as normal or abnormal based on the presence of DR.

\subsection{Database}

The retinal fundus images of the diabetic patients are collected from the Government hospital, which are taken by the digital fundus camera. The database contains 60 images of the retina, and was saved in JPEG format. These 60 images were divided into a training set and a testing set. The database used here is diagnosed by the medical experts for each image such as Diabetic Retinopathy and normal image. 
Recent Advances in Communication, Electronics \& Electrical Engineering

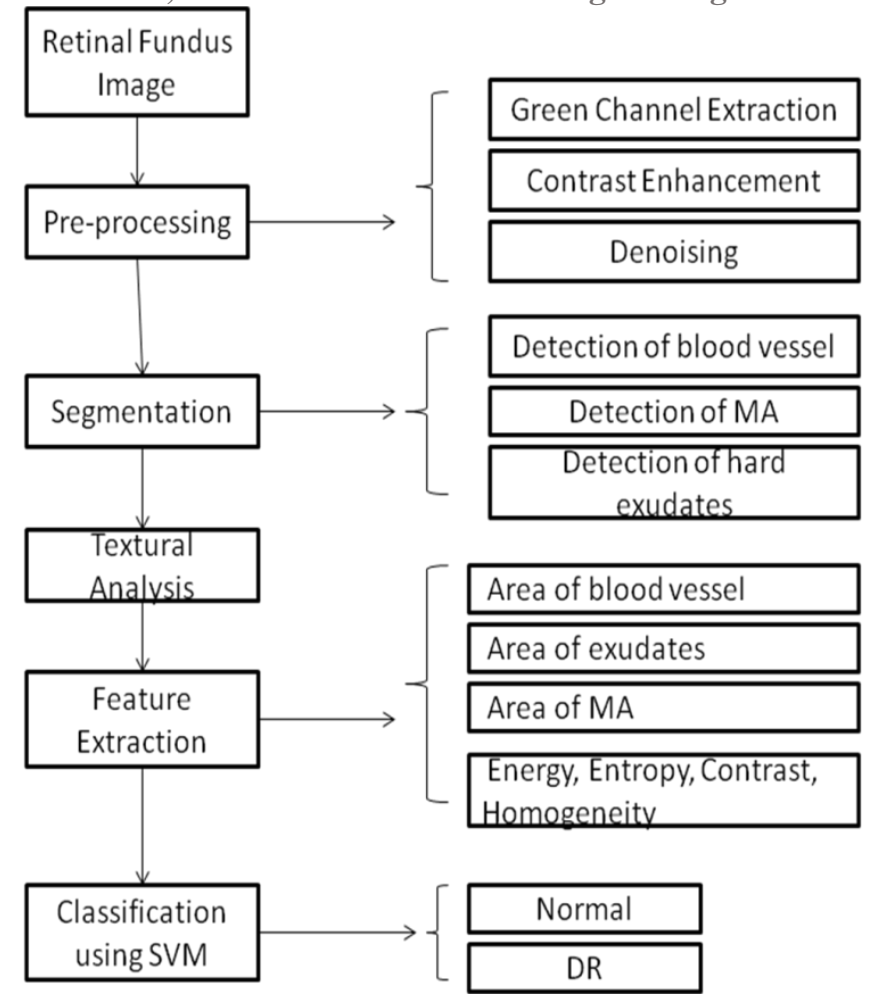

Figure 2: Proposed methodology

\subsection{Pre-Processing}

Green channel extraction: Green Channel Extraction is the process of converting the color image into green channel image. Normally a RGB color consists of red, green and blue color. The green channel extraction produces high contrast image. The green color plane was used in this analysis since it shows the best contrast between the vessels and the background retina in the eye [1, 4, and 7].

Contrast Enhancement: Adaptive histogram equalization is an image processing technique used to improve contrast in images. It is considered an image enhancement technique capable of improving an image's local contrast, bringing out more detail in the image [4,5].

Denoising: It is usually necessary to perform a high degree of noise reduction in an image before performing higher level processing steps. Median filter is a non-linear digital filtering technique which is often used to eliminate noise from images or other signals. Its edge-conserving nature makes it useful in cases where edge blurring is undesirable. Median filtering operation replaces a pixel by the median of all pixels in the neighborhood of small sliding window. It may be applied prior to segmentation in order to reduce the amount of noise in a stack of 2D images. The median filter reduces the variance of the intensities in the image. Median filters change the intensity mean value of the image, if the spatial noise distribution in the image is not symmetrical within the window $[8,10]$. 


\subsection{Segmentation of Retinal Structures}

Detection of Blood Vessels: The segmentation of blood vessels is done by using the Kirsch's edge detection and morphological method. This is followed by applying Kirsch's edges [11]. Kirsch's edges helps to capture the edges of the exudates. This edge detector is based on the kernel $\mathrm{k}$ evaluated at 8 different directions on the image. The kernel is given by,

$$
k=\left[\begin{array}{ccc}
5 / 15 & -3 / 15 & -3 / 15 \\
5 / 15 & 0 & -3 / 15 \\
5 / 15 & -3 / 15 & -3 / 15
\end{array}\right]
$$

The kernel outputs are combined together by selecting the maximum value found on each pixel output. This is followed by erosion operation and binary conversion.

Detection of Exudates and Microaneurysms: Morphological operators are used for the detection of exudates and MA in this work. The morphological operators extract relevant structures of the image by probing the image with another set of known shape called structuring element (SE) [7]. By using the prior knowledge of the geometry the shape of the SE is chosen. The morphological operations are erosion, dilation, opening, closing. Erosion computes the minimum of each pixel's neighborhood and is given by,

$$
A \Theta B=\left\{\left\langle z \in E \mid B_{z} \subseteq A\right\rangle\right\}
$$

Where $\mathrm{A}$ is a binary image, $\mathrm{B}$ is a structuring element and $\mathrm{E}$ is the Euclidean space.

Dilation computes the maximum of each pixel's neighborhood and is given by,

$$
A \oplus B=\left\{\left\langle z \in E \mid\left(B^{s}\right)_{\approx}\right\rangle \cap A \neq \phi\right\}
$$

Opening involves single erosion followed by a single dilation by the same operator.

$$
A \circ B=(A \Theta B) \oplus B
$$

Closing involves a single dilation followed by single erosion by the same operator.

$$
A \bullet B=(A \oplus B) \Theta B
$$

The steps for the detection of exudates are as follows. Green channel of the retinal image is extracted from the colored image. First median filtering is applied to the green image. Then morphological reconstruction is done to obtain the background image, which is subtracted from the original image. Next step is to remove all the connected components form the binary image that has fewer than P pixels so that the resultant binary image will contain only optic disc, where $\mathrm{P}$ was chosen such that it is smaller than the maximum size of optic disc. The optic disc occupies nearly $80 \times 80$ pixels. The resulting image that contains only the optic disc is subtracted from the threshold image so that it delivers an image that contains only exudates. 
Recent Advances in Communication, Electronics \& Electrical Engineering

Microaneurysms are also detected using morphological operators in this work. The first step is adaptive histogram equalization for image enhancement. Closing operator is applied to eliminate the details. Then filling operation is done to fill the holes in the vessels. Then the difference between the closed image and the filled image is found.

$$
f_{\text {diff }}=\operatorname{close}(f)-\operatorname{fill}(f)
$$

This difference image is binarized by thresholding. Then extended-minima transform is applied to this binary image. The extended-minima transform is the regional minima of h-minima transform. This is a thresholding technique that brings most of the valleys to zero. The h-minima transform suppresses all the minima in the intensity image whose depth is less than or equal to a predefined threshold. In the output binary image (fE), the white pixels represent the regional minima in the original image. Regional minima are connected pixels with the same intensity value, whose external boundary pixels all have a higher value. Finally the vessels and exudates are removed to separate out the MAs.

$$
f_{M A}=f_{E}-f_{v}-f_{e x}
$$

\subsection{Texture Analysis}

Texture means repeating patterns of local variation of pixel intensities. It gives information about the arrangement of surface pixels and their relationship with the surrounding pixels. Statistical texture analysis is based on Gray Level Co-occurrence Matrix (GLCM). It is a tabulation of how often different combinations of pixel grey levels occur in an image. For a 2-dimensional image $\mathrm{f}(\mathrm{x}, \mathrm{y})$ with $\mathrm{N}$ grey levels, the GLCM $p(d, \phi)$ for each $d$ and $\phi_{\text {is given by, }}$

$$
p(d, \phi)=\left|\begin{array}{ccccc}
p_{0,0} & p_{0,1} & \cdot & \cdot & p_{0, N-1} \\
p_{1,0} & p_{1,1} & \cdot & \cdot & p_{1, N-1} \\
\cdot & \cdot & \cdot & \cdot & \cdot \\
\cdot & \cdot & \cdot & \cdot & \cdot \\
p_{N-1,0} & p_{N-1,1} & \cdot & \cdot & p_{N-1, N-1}
\end{array}\right|
$$

$p_{i, j}=\frac{\text { number of pixel pairs with int ensity }(i, j)}{\text { total number of pairs considered }}$

$p_{i j}$ is defined as the relative number of times gray level pair (i,j) occurs when pixels separated by the distance $\mathrm{d}$ along the angle $\phi$ are compared. Each element is normalized by the total number of occurrences to form the GLCM P. The commonly extracted textural features from GLCM are contrast, homogeneity, correlation and energy $[7,10]$. 
Recent Advances in Communication, Electronics \& Electrical Engineering

\subsection{Feature Extraction}

The features are extracted for the classification purpose, which are obtained from segmentation of retinal structures and texture analysis. These features are the area of blood vessels, area of exudates, area of MA, contrast, homogeneity, correlation and energy.

Area of blood vessels: It is determined by finding the total number of white (vessel) pixels in the vessel-segmented image.

Area of exudates: It is determined by finding the total number of white pixels in the exudates image.

Area of MA: It is determined by finding the total number of white pixels in the MA image.

Contrast: It is a measure of the intensity contrast between a pixel and its neighbor over the whole image, and is given by,

contrast $=\sum_{i=0}^{N-1} \sum_{j=0}^{N-1}(i-j)^{2} p_{i j}$

Where ${ }^{p_{i j}}$ are the elements of the GLCM.

Homogeneity: It measures the closeness of the distribution of elements in the GLCM to the GLCM diagonal and can be mathematically written as

$$
\text { Homogeneity }=\sum_{i=1}^{N-1} \sum_{j=0}^{N-1} \frac{p_{i j}}{(1+|i-j|)}
$$

Correlation: It calculates the linear dependency of the gray level values in the co-occurrence matrix. It is represented mathematically as

$$
\text { Correlation }=\sum_{i=0}^{N-1} \sum_{j=0}^{N-1} \frac{\left(i-\mu_{i}\right)\left(j-\mu_{j}\right) p_{i j}}{\sigma_{i} \sigma_{j}}
$$

Where $\mu \mathrm{i}, \mu \mathrm{j}, \sigma \mathrm{i}$ and $\sigma \mathrm{j}$ are the means and standard deviations of $\mathrm{Pi}$ and $\mathrm{Pj}$.

Energy: It is the sum of squared elements in the co-occurrence matrix.

$$
\text { Energy }=\sum_{i=0}^{N-1} \sum_{j=0}^{N-1} p_{i j}^{2}
$$

All the seven features together form a feature vector of an image.

\subsection{Classification}

In this work, classification based on the extracted features is done by using SVM classifier [5, 7, 8 , and 10]. SVM is used to classify the group of eye images as either affected or normal 
Recent Advances in Communication, Electronics \& Electrical Engineering depending on the feature values. A Support Vector Machine (SVM) performs classification by constructing an $\mathrm{N}$ dimensional hyper plane that optimally separates the data into two categories.

Based on the results of area Computation and texture analysis, the system will classify the image as normal or diabetic retinopathy. In the normal stage, there are no micro aneurysms and exudates. In DR stage, both exudates and micro aneurysms are present.

\section{Results and Discussions}

The study evaluating a new test that screens people for a disease. Each person taking the test either has or does not have the disease. The test outcome can be positive (predicting that the person has the disease) or negative (predicting that the person does not have the disease). The test results for each subject may or may not match the subject's actual status. In that setting:

True positive: Affected retina correctly diagnosed as defected; False positive: Healthy retina incorrectly identified as affected; True negative: Healthy retina correctly identified as healthy False negative: Affected retina correctly identified as healthy

In general, Positive $=$ identified and negative $=$ rejected. Therefore, True positive $=$ correctly identified; False positive = incorrectly identified; True negative = correctly rejected False negative $=$ incorrectly rejected

The screening diabetic retinopathy is performed with respect to sensitivity and specificity.

$$
\begin{aligned}
\text { sensitivity } & =\frac{\text { number of true positives }}{\text { number of true positives }+ \text { number of true negatives }} \\
& =\frac{\text { number of true positives }}{\text { total number of sick individuals in population }} \\
& =\text { probability of a positivetest given that the patient is ill } \\
\text { specificiy } & =\frac{\text { number of true negatives }}{\text { number of true negatives }+ \text { number of false positives }} \\
& =\frac{\text { number of true negatives }}{\text { total number of well individuals in population }} \\
& =\text { probability of a negative test given that the patient is well }
\end{aligned}
$$


Recent Advances in Communication, Electronics \& Electrical Engineering

\subsection{Simulation Results of MATLAB}

\subsubsection{Segmentation of Blood Vessels}

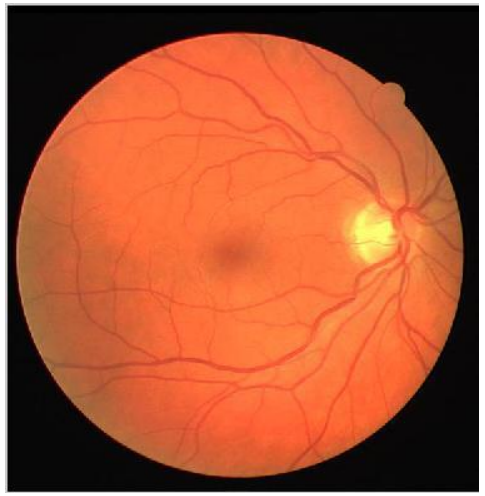

a)

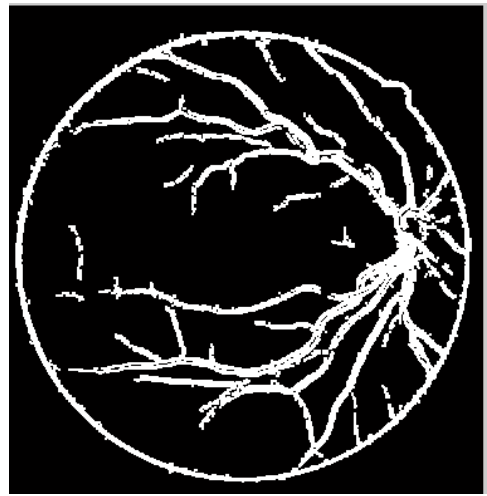

d)

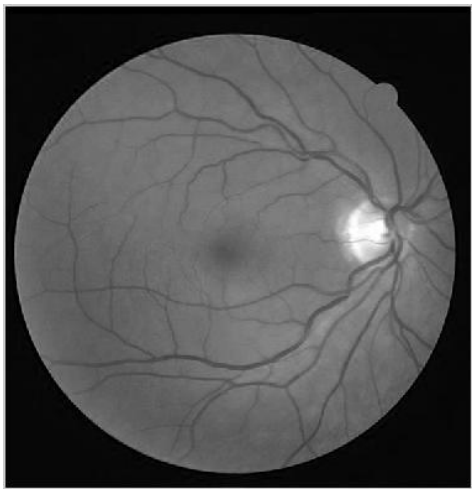

b)

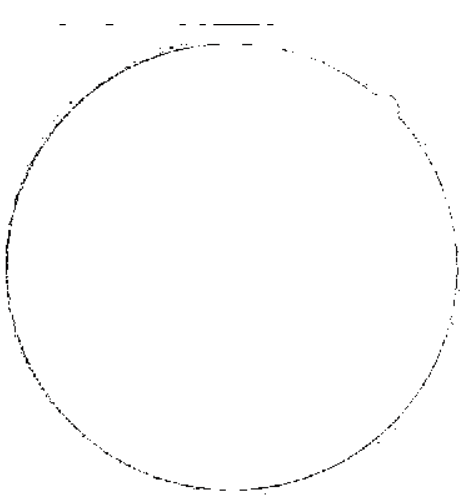

e)

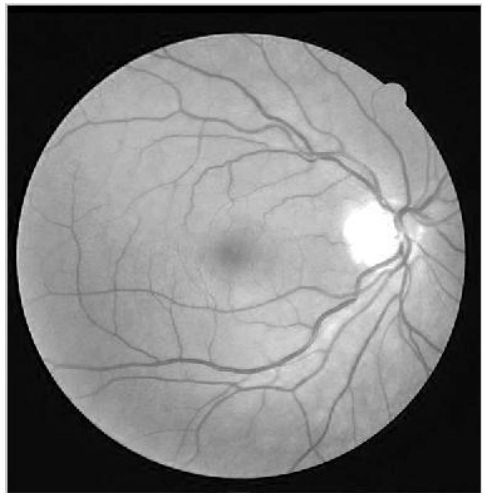

c)

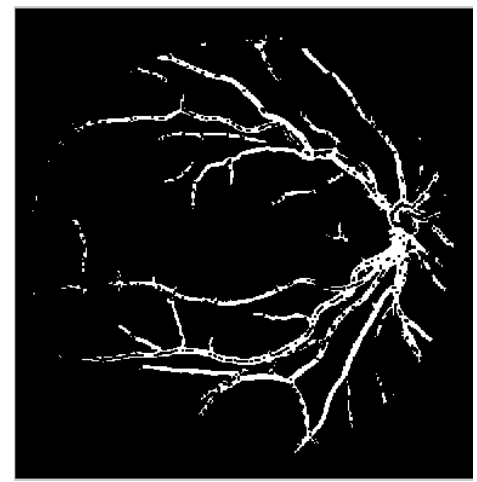

f)

Figure 3: (a) retina image (b) green channel image (c) contrast enhancement (d) kirsch's edge detection (e) mask creation (f) segmented blood vessels

The segmentation of blood vessels is done by using Kirsch's edge detection (method 1) and morphological operation, which is shown in Fig 3.

\subsubsection{Segmentation of Exudates}

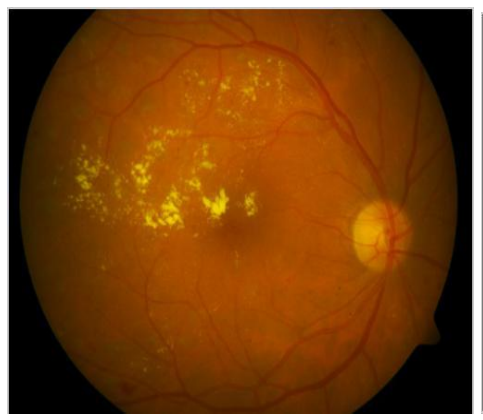

a)

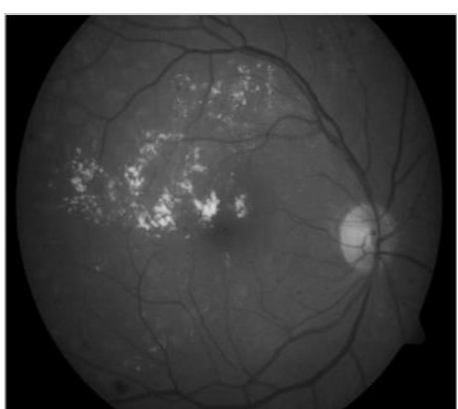

b)

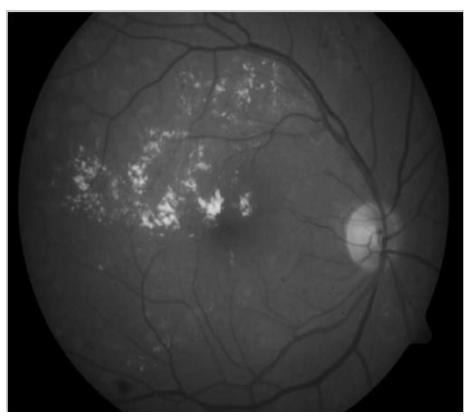

c) 
Recent Advances in Communication, Electronics \& Electrical Engineering

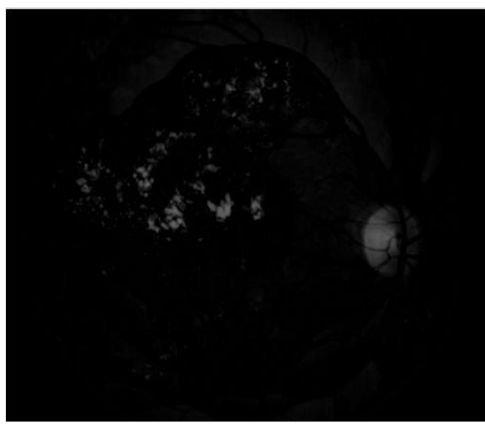

d)

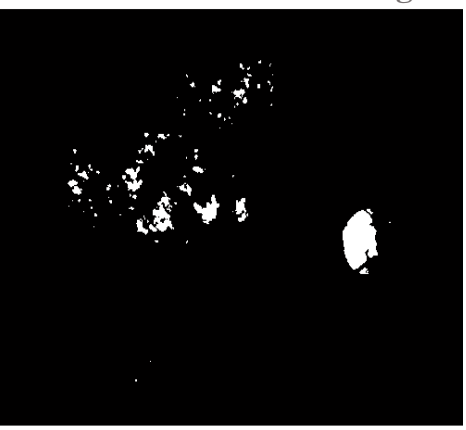

e)

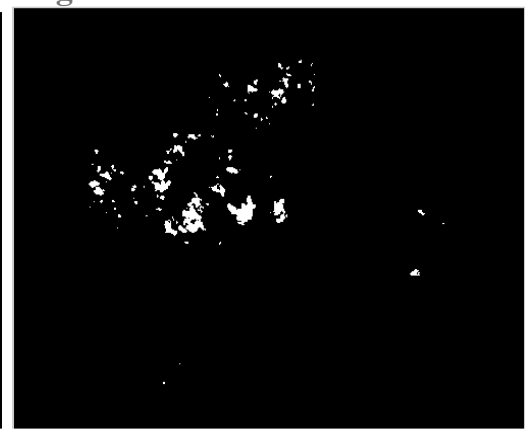

f)

Figure 4: (a) retina image (b) green channel image (c) median filtering (d) background image (e) exudates and optic disc (f) extracted exudates

\subsubsection{Segmentation of Microaneurysms}

Microaneurysms are segmented using extended minima transform and morphological operations in this work.

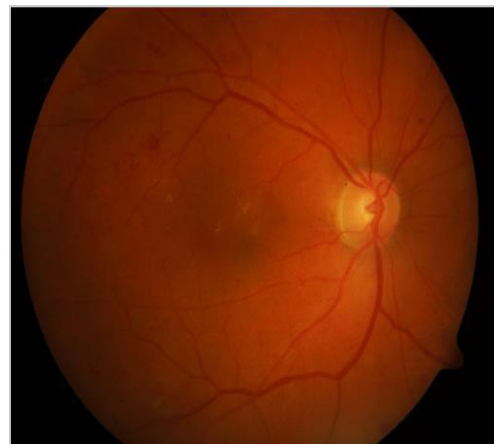

a)

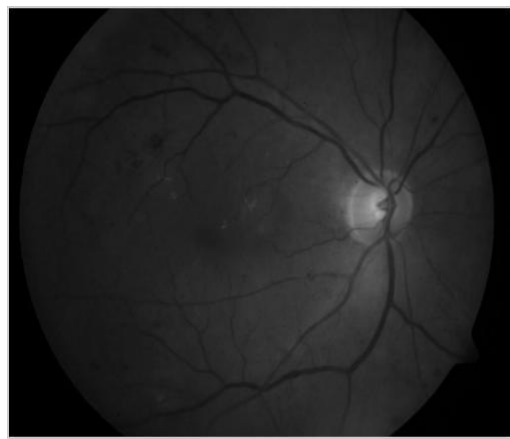

a)

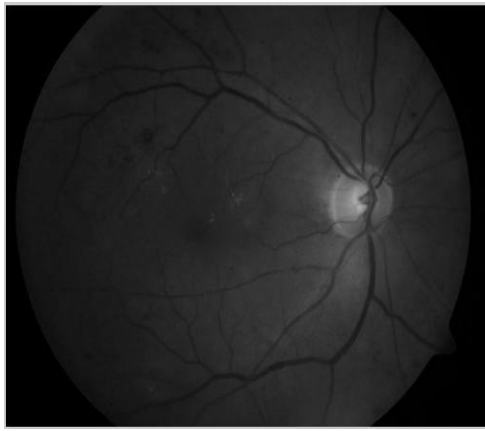

b)

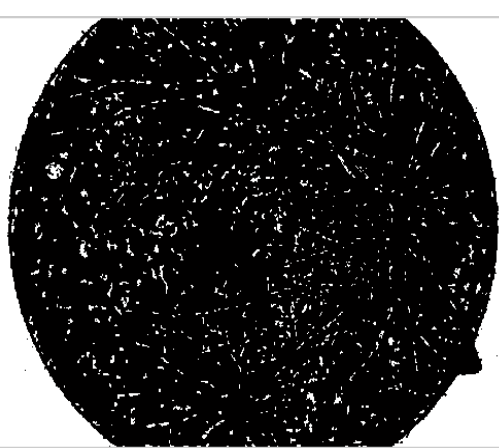

b)

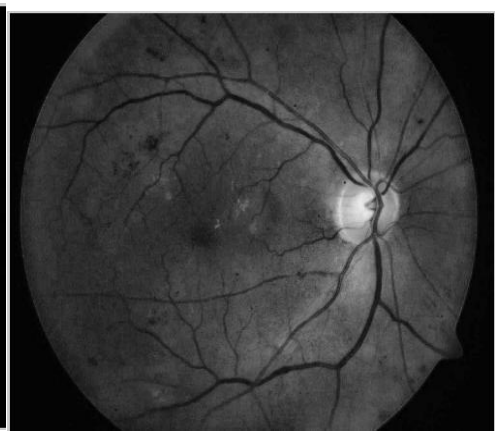

c)

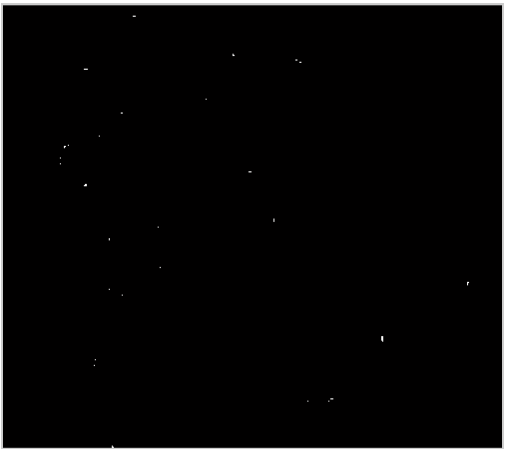

c)

Figure 5: (a) retina image (b) green channel image (c) contrast enhancement (d) median filtering (e) extended minima image (f) segmented microaneurysms 
Recent Advances in Communication, Electronics \& Electrical Engineering

\subsection{Simulation Result in LabView}

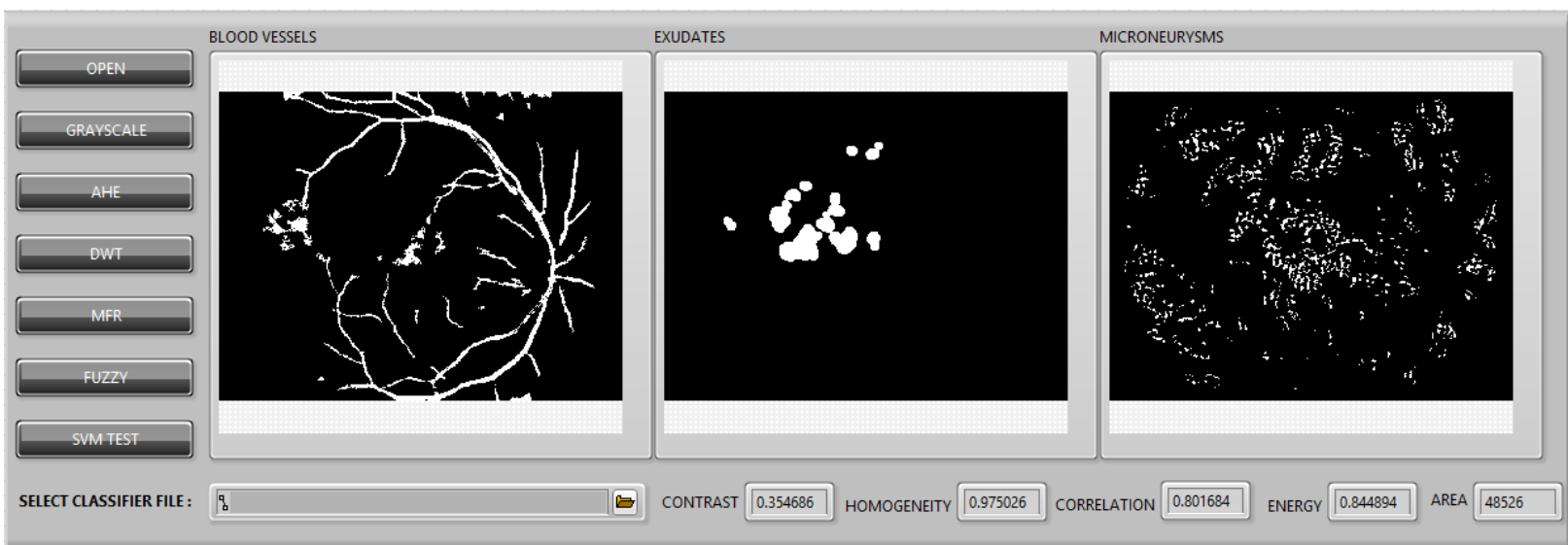

Figure 6: Snap shot of retina after which showing segmentation of blood vessels using method 2, exudates, microaneurysms and extracted feature values

\subsection{Classification Result}

\subsubsection{Using MATLAB}

All the features are extracted and combined to a single vector and this vector is used as input to the SVM classifier. The image is classified as 'Normal' if it does not contain any exudates and microaneurysms. The image is classified as 'Moderate' if it contained only microaneurysms and not the exudates. The image is classified as 'Severe' if it contains both microaneurysms and exudates.

Table 1: Classification results in Matlab

\begin{tabular}{|l|l|l|l|}
\hline Class & No. of Training Images & No. of Testing Images & No. of Correctly classified Images \\
\hline Normal & 100 & 14 & 13 \\
\hline Mild & 100 & 14 & 12 \\
\hline Severe & 100 & 14 & 12 \\
\hline
\end{tabular}

True Positive $=12$, False Positive $=1$, True Negative $=13$, False Negative $=2$ Sensitivity $=\mathrm{TN} /(\mathrm{TN}+\mathrm{FP})=92.85 \%$ Specificity $=\mathrm{TP} /(\mathrm{TP}+\mathrm{FN})=85.71 \%$

\subsubsection{Using LabView}

Table 2: Classification results in LabView

\begin{tabular}{|l|l|l|l|}
\hline Class & No. of Training Images & No. of Testing Images & No. of Correctly classified Images \\
\hline Normal & 100 & 14 & 13 \\
\hline Mild & 100 & 14 & 10 \\
\hline Severe & 100 & 14 & 11 \\
\hline
\end{tabular}

True Positive $=13$, False Positive $=1$, True Negative $=12$, False Negative $=2$

Sensitivity $=\mathrm{TN} /(\mathrm{TN}+\mathrm{FP})=92.85 \% ;$ Specificity $=\mathrm{TP} /(\mathrm{TP}+\mathrm{FN})=78.52 \%$ 
Recent Advances in Communication, Electronics \& Electrical Engineering

The main objective of LabView simulation tool is that it allows easy implementation on the LabView FPGA tool kit. Due to this the specifications used are different compared to the Matlab which makes the blocks more compatible to be implemented in the hardware. Different parameters for individual block sets are present in LabView, so that classification accuracy of the SVM classifier varies for LabView and Matlab.

\section{Conclusion}

Diabetic Retinopathy system consists of three modules, Segmentation, feature extraction and classification is developed and tested using simulation tools Matlab and LabView. Segmentation module includes segmentation of blood vessels, exudates and microaneurysms. Feature extraction includes extracting different features from GLCM and then classification using SVM.

Kirsch's edge detection and matched filter response are used for blood vessel segmentation. Exudates are segmented using image reconstruction and connected component analysis. Microaneurysms are segmented by using extended minima transform. The features are extracted by textural analysis. The extracted features describe the relevant information from the input data in order to perform the identification process. The extracted features are used for effective classification of three different stages of diabetic retinopathy that is normal, moderate or severe. The accuracy of classification is calculated in terms of sensitivity and specificity.

This system intends to help in the screening process to detect symptoms of Diabetic Retinopathy. This can be a preliminary diagnosis tool or decision support system for ophthalmologists. The proposed technique does not require any user intervention, and has consistent performance in both normal and abnormal images.

\section{References}

[1] Iqbal.M.I, Aibinu.A.M, Gubbal.N.S and Khan.A "Automatic diagnosis of Diabetic Retinopathy using fundus images”, Ph.D. Dissertation, Blekinge Institute of Technology, 2006. MEE06:19, october 2006.

[2] Jestin V.K, Anitha J and Jude Hemanth, "Texture feature extraction for retinal image processing", in Proc. International Conference on Computing, Electronics and Electrical Technologies (ICCEET), vol. 1, pp: 548-551, 2012.

[3] ManjulaSriRayudu, Vaibhav Jain and MM.RaoKunda , "Review of image processing techniques for automatic detection of eye diseases", in Proc. Sixth International conference on sensing technology (ICST), pp: 320-325, 2012.

[4] Priya.R, Aruna.P, "Review of automated diagnosis of diabetic retinopathy using the support vector machine", International journal of applied engineering research, vol. 1, no 4, pp: 844-863, 2011.

[5] DRIVE Database

Retrieved from http://www.isi.uu.nl/Research/Database/DRIVE, Accessed on 02-01-2014.

[6] DIARETDB1 Database

Retrieved from http://www2.it.lut.fi/project/imagetert/diaretdb1/, Accessed on 02-01-2014

[7] DIARETDB1 Database

Retrieved from http://www2.it.lut.fi/project/imagetert/diaretdb0/, Accessed on 02-01-2014 
Recent Advances in Communication, Electronics \& Electrical Engineering

[8] Istvan Lazar and AndrasHajdu, "Retinal microaneurysm detection through local rotating crosssection profile analysis", IEEE transaction on medical imaging, vol. 32, no. 2, pp: 400-407, 2013.

[9] Giribabukande, P.Venkatasubbaiah, T.sathyasavithri, "Feature extraction in Digital fundus Image", Journal of Medical and Biological engineering vol. 29, no. 3, pp 122-130, 2009.

[10] CemalKose, UgurSevik, CevatIkibas and HidayetErdol, "Simple methods for segmentation and measurement of diabetic retinopathy lesions in retinal fundus images", in Proc. Computer Methods and Programs in Biomedicine (CMPB), vol. 107, no. 2, pp: 274-293, 2012.

[11] A.Sopharak,B.Uyyanonvara,S.Barman and T.H.Williamson, "Automatic detection of diabetic retinopathy exudates from non-dilated retinal images using mathematical morphology methods", in Proc. Computerized Medical Imaging and Graphics ,vol. 32, pp: 720-727, 2009.

[12] RagavVenkatesan, ParagChandakkar, Baoxin Li, Senior member, IEEE, and Helen K. Li, "Classification of diabetic retinopathy images using multi-class multi instance learning based on colorcorrelogram features", in Proc. 34th International conference of IEEE EMBS, pp: 14621465, 2012.

[13] Dr.Chandrashekar. M. Patil, "An Approach for the Detection of Vascular Abnormalities in Diabetic Retinopathy", International Journal of Data Mining Techniques and Applications, vol. 02, no. 5, pp. 246-250, 2013.

[14] Mahendran.G, Dhanashekar.R, NarmadhaDevi.K.N, "Recognition of Retinal Exudates for Diabetic Retinopathy and its Severity Level Assessment" IJECEAR, vol. 2, no. 1, pp: 104-108, 2014.

[15] Selvathi.D, N.B.Prakash, NeethiBalagopal,"Automatic Detection of Diabetic Retinopathy for Early Diagnosis using Feature Extraction and Support Vector Machine", International Journal of Emerging Technology and Advanced Engineering, vol. 2, no. 11, pp: 103-108, 2012.

[16] Berrichi Fatima Zohra, Benyettou Mohamed, "Automated diagnosis of retinal images using the Support Vector Machine(SVM)", Laboratoire de Modélisation et Optimisation des SystèmesIndustriels : LAMOSI, pp: 1-6, 2011.

[17] Tadej Tasner, Darko Lovrec, Francisek Trsner and Jorg Edler, "Comparison of LabVIEW and MatLab for scientific research", International journal of engineering, vol. 3, no.7, pp: 133-137, 2012.

\footnotetext{
*Corresponding author.

E-mail address: purushothama.t.1@gmail.com
} 